\title{
Lactobacillus rhamnosus GG and Saccharomyces cerevisiae boulardii exert synergistic antipathogenic activity in vitro against enterotoxigenic Escherichia coli
}

\author{
F. Moens ${ }^{1}$, C. Duysburgh ${ }^{1}$, P. van den Abbeele ${ }^{1}$, M. Morera ${ }^{2}$ and M. Marzorati ${ }^{1,3^{*}}$ \\ ${ }^{1}$ ProDigest bvba, Technologiepark 82, 9052 Ghent, Belgium; ${ }^{2}$ Ipsen Pharma SAS, 65 Quai George Gors, 92650 Boulogne \\ Billancourt Cedex, France; ${ }^{3}$ Center of Microbial Ecology and Technology (CMET), Ghent University, Coupure Links 653, \\ 9000 Ghent, Belgium; massimo.marzorati@prodigest.eu
}

Received: 9 April 2019 / Accepted: 23 July 2019

(c) 2019 Wageningen Academic Publishers

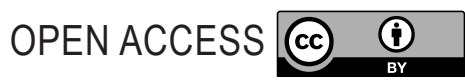

RESEARCH ARTICLE

\begin{abstract}
Short-term colonic in vitro batch incubations were performed to elucidate the possible synergistic effects of Lactobacillus rhamnosus GG (CNCM-I-4798) and Saccharomyces cerevisiae boulardii (CNCM-I-1079) (associated in Smebiocta/Smectaflora Protect ${ }^{\oplus}$ ) on the colonic microbial fermentation process, as well as their antipathogenic activity against enterotoxigenic Escherichia coli (LMG2092) (ETEC). These incubations adequately simulate the native microbiota and environmental conditions of the proximal colon of both adult and toddler donors, including the colonic mucosal layer. Results indicated that both strains were capable of growing together without showing antagonistic effects. Co-cultivation of both strains resulted in increased butyrate (stimulated by L. rhamnosus GG), propionate (stimulated by S. boulardii), and ethanol (produced by S. boulardii) production compared to the control incubations, revealing the additive effect of both strains. After inoculation of ETEC under simulated dysbiotic conditions, a 40 and $46 \%$ reduction in the concentration of ETEC was observed upon addition of both strains during the experiments with the adult and toddler donor, respectively. Furthermore, ETEC toxin levels decreased upon $S$. boulardii inoculation, probably due to proteolytic activity of this strain, with a synergistic effect being observed upon co-cultivation of L. rhamnosus GG and S. boulardii resulting in a reduction of 57 and $46 \%$ for the adult and toddler donor, respectively. Altogether, the results suggest that both probiotics together may help microbiota functionality, in both adults and toddlers and under healthy or impaired conditions, which could be of great interest when the colonic microbiota is dysbiotic and therefore sensitive to pathogenic invasion such as during antibiotic treatment.
\end{abstract}

Keywords: LGG, yeast, ETEC, gut microbiota, dysbiosis

\section{Introduction}

Probiotics are recognised for their role in improving human health, most likely by modifying the gut microbiota composition in a transient manner. Indeed, probiotics are defined as live microorganisms which when administered in adequate amounts confer a health benefit on the host (Hill et al. 2014). Among the most documented probiotic strains, Lactobacillus rhamnosus GG (Doron et al., 2005) has been associated with several health-promoting properties including prevention of antibiotic-induced diarrhoea (Vanderhoof et al., 1999), recovery from disease-related types of diarrhoea (Guandalini et al., 2000; Szajewska et al., 2011), prevention of atopic disease (Kalliomaki et al., 2001), improvement of immune function (Pena and Versalovic, 2003; Pessi et al., 2000) and antipathogenic activity (De Keersmaecker et al., 2006) due to the production of antimicrobial compounds. For example, the antimicrobial activity of $L$. rhamnosus GG against the intestinal pathogen Salmonella typhimurium has been linked to lactic acid secretion into the culture medium (De Keersmaecker et al., 2006). Another group investigating the antipathogenic effect of $L$. rhamnosus $\mathrm{GG}$ against the respiratory tract pathogen Moraxella catarrhalis obtained similar results (Van den Broek et al., 2018) Another extensively used healthpromoting microorganism is the non-pathogenic yeast Saccharomyces cerevisiae boulardii. Yeasts are resistant to antibiotics and therefore are frequently used as preventive 
and therapeutic agents for antibiotic-associated diarrhoea (Czerucka et al., 2007). Furthermore, S. boulardii has shown excellent survival through the upper gastro-intestinal tract, which is an important characteristic of probiotic products intended for oral consumption (Tompkins et al., 2011). Several mechanisms of action have been described for the health-promoting activity of $S$. boulardii, including regulation of intestinal microbial homeostasis, regulation of immune response, improvement of gut barrier function, antitoxin effects and antimicrobial activity (Kelesidis and Pothoulakis, 2012). S. boulardii has been shown to exert antipathogenic activity against several disease-causing Escherichia coli strains, such as enterohemorrhagic $E$. coli (EHEC) (Dahan et al., 2003; Dalmasso et al., 2006), enteropathogenic E. coli (EPEC) (Czerucka et al., 2000) and enterotoxigenic E. coli (ETEC) (Badia et al., 2012).

ETEC infection is recognised as one of the most common causes of traveller's diarrhoea and of childhood diarrhoea in developing countries (Anonymous, 2006). Furthermore, foodborne outbreaks of ETEC infection have emerged in developed countries, such as the USA (Dalton et al., 1999), Norway (MacDonald et al., 2015), Denmark (Pakalniskiene et al., 2009) and South Korea (Shin et al., 2016). One of the major virulence factors of ETEC infection includes adherence of the pathogenic strain to the epithelium of the small intestine, which is mediated by several colonisation factors including numerous fimbriate surface antigens (Gaastra and Svennerholm, 1996). Colonisation of the small intestine is followed by the release of either or both heat-stable and heat-labile enterotoxins, causing diarrhoea (Qadri et al., 2005). The resulting infection generally lasts for several days and ranges from illness with mild diarrhoea to severe cholera-like disease (Anonymous, 2006). Several studies have reported the potential role of probiotics in the prevention and/or treatment of ETEC infections (Roselli et al., 2006; Tsai et al., 2008). L. rhamnosus GG was shown to partially impair ETEC adhesion to intestinal epithelial cells in vitro, probably through competition for binding sites and/or secretion of a non-proteinaceous antimicrobial metabolite (Roselli et al., 2006). Additionally, L. rhamnosus GG exerted anti-inflammatory properties in response to ETEC-induced inflammation by regulation of chemokine and cytokine expression. Furthermore, $S$. boulardii was shown to prevent ETEC infection in vitro by preventing ETEC from adhering to intestinal epithelial cells and reducing pathogenic inflammation via secretion of anti-inflammatory factors (Badia et al., 2012). As such, co-administration of L. rhamnosus GG together with $S$. boulardii could therefore potentiate the beneficial effects of these probiotics on ETEC infection.

Further, since most studies regarding the interactions of probiotics with ETEC have been performed in vitro and focused on the small intestinal environment, only a limited amount of data exists on the interactions of ETEC with the colonic microbiota and probiotics (Roussel et al., 2017a,b). In humans, the colonic microbiota plays a predominant role on the possible invasion of ETEC and induction of disease (Pop et al., 2016). Indeed, microbiota compositional analysis revealed an association between the presence of 12 Operational Taxonomic Units (OTUs) in the microbiota and the absence of development of diarrheal disease upon ETEC challenge. Similar results were obtained during studies with piglets challenged with ETEC (Bin et al., 2018). Microbiome profiling has revealed that travellers who develop diarrhoea upon ETEC infection have a dysbiotic colon microbiota that is characterised by a high Firmicutes:Bacteroidetes ratio (Youmans et al., 2015). As such, modulation of the gut microbiota and its associated fermentation process through the ingestion of probiotics could be used as a strategy to protect against ETEC infection. Indeed, L. rhamnosus GG has been shown effective at preventing post-weaning diarrhoea in piglets, which was associated with modulation of the intestinal microbiota (Zhang et al., 2010). Modulation of the gut microbiota in newly weaned pigs through a select mixture of Bacillus species has been shown to improve ETECinduced enteritis symptoms (Zhang et al., 2017). Finally, pre-supplementation of the probiotic strain Lactobacillus reuteri $\mathrm{HCM} 2$ to mice prevents the dysbiosis of the colonic microbiota caused by ETEC through modulation of the gut microbial community (Wang et al., 2018).

The main objective of this study was to determine how L. rhamnosus GG and S. boulardii, when administered together, would affect the colonic microbial fermentation process and identify their respective and combined activity against ETEC. We hypothesised that these strains could possibly act synergistically when added together to a healthy and altered colonic microbiota environment in vitro.

\section{Materials and methods}

\section{Chemicals and test products}

All chemicals were obtained from Sigma-Aldrich (Overijse, Belgium) unless stated otherwise. Lallemand Health Solutions Inc. (Blagnac, France) provided the test strains Saccharomyces cerevisiae boulardii (CNCM-I-1079) and Lactobacillus rhamnosus GG (CNCM-I-4798) associated in Smebiocta/Smectaflora Protect ${ }^{\oplus}$. Enterotoxigenic Escherichia coli LMG2092 (ETEC) was obtained from BCCM/LMG (Ghent, Belgium).

\section{Short-term colonic microbiota batch incubations}

To account for inter-individual differences, two different donors, an adult and a toddler, were used as a source of colonic background community. Under healthy conditions for both donors, the short-term colonic batch incubations were performed in $120 \mathrm{ml}$ penicillin bottles and initiated 
by the addition of $63 \mathrm{ml}$ colonic background medium containing host- and diet-derived nutritional compounds. Four types of colonic incubations were performed. During the control (CTRL) incubations only the colonic background microbiota was added. During the 'LGG' experiments $10^{9}$ cfu of L. rhamnosus GG were dosed to the incubations together with the colonic background microbiota. During the 'SB' experiments $50 \mathrm{mg}$ of $S$. boulardii powder was added to the colonic incubations containing a metabolically active colonic microbiota. Both L. rhamnosus GG, S. boulardii and a colonic background microbiota were dosed to the 'LGG + SB' incubations. Background microbiota were obtained from both a healthy adult donor and from a healthy 3-year old toddler donor. Briefly, faecal samples were freshly collected in containers containing an AnaeroGen sachet to maintain anaerobic atmosphere. A faecal inoculum was prepared by making a $1: 13(\mathrm{w} / \mathrm{v})$ mixture of the faecal sample with anaerobic phosphate buffer $\left(\mathrm{K}_{2} \mathrm{HPO}_{4} 8.8 \mathrm{~g} / \mathrm{l} ; \mathrm{KH}_{2} \mathrm{PO}_{4}\right.$ $6.8 \mathrm{~g} / \mathrm{l}$; sodium thioglycolate $0.1 \mathrm{~g} / \mathrm{l}$; sodium dithionite $0.015 \mathrm{~g} / \mathrm{l}$ ). After homogenisation (10 min, BagMixer ${ }^{\circledR} 400$, Interscience, Louvain-La-Neuve, Belgium) and removal of big particles via centrifugation ( $2 \mathrm{~min}, 500 \times g$ ), an inoculum corresponding to $10 \%(\mathrm{v} / \mathrm{v})$ was added to the different incubations. Furthermore, during all incubations the mucosal layer of the colon was simulated by the addition of mucus beads (Van den Abbeele et al., 2012). Briefly, mucus beads were prepared by covering microcosms (Kaldness K1, AnoxKaldnes AB, Lund, Sweden) with a mucin agar solution and combining them in a polyethylene netting (Zakkencentrale, Rotterdam, the Netherlands) at a ratio of 1 microcosm per $9 \mathrm{ml}$ of colonic medium. All incubations were performed under strict anaerobic conditions. Anaerobiosis was obtained by flushing with $\mathrm{N}_{2}$. All experiments were incubated at $37{ }^{\circ} \mathrm{C}$ for a period of $48 \mathrm{~h}$ under continuous mixing ( $90 \mathrm{rpm}$ ). All experiments were performed in biological triplicate.

Under dysbiotic conditions, colonic incubations were performed in a similar way, with the following modifications: during all incubations, only $0.02 \%(\mathrm{v} / \mathrm{v})$ of background colonic microbial community was added in order to simulate dysbiosis. Furthermore, $2 \%(\mathrm{v} / \mathrm{v})$ of an overnight ETEC culture was added to all incubations, resulting in approximately $7.5 \mathrm{log}$ ETEC/ml at the start of the incubations. ETEC was grown overnight in nutrient broth at $37{ }^{\circ} \mathrm{C}$ under normal atmospheric conditions and continuous shaking (110 rpm). The overnight culture was subsequently centrifuged at $1,500 \times g$ for $10 \mathrm{~min}$, the supernatant was removed and the pellet was resuspended in sterile phosphate-buffered saline (PBS).

\section{Microbial growth}

Quantification of the viable microbial cells of $L$. rhamnosus GG, S. boulardii, and ETEC during the colonic batch incubations was determined by generating cell pellets at different time points during the incubation (baseline, $6 \mathrm{~h}, 24 \mathrm{~h}$, and $48 \mathrm{~h}$ ) which were subjected to propidium monoazide (PMA, Biotium, Hayward, CA, USA) treatment. Briefly, $50 \mu \mathrm{l}$ of sample was added to $450 \mu \mathrm{l}$ of sterile anaerobic peptone water. PMA was added to reach a final concentration of $50 \mu \mathrm{M}$. Samples were shaken in the dark for $5 \mathrm{~min}$ at room temperature. Afterwards the PMAtreated cell suspensions were placed in a LED-Active Blue system (Ingenia Biosystems, Barcelona, Spain) for $15 \mathrm{~min}$ followed by centrifugation for $5 \mathrm{~min}$ at 7,690×g. Cell pellets were stored at $-20{ }^{\circ} \mathrm{C}$. Subsequently, DNA was extracted from these PMA-treated cells using the DNeasy UltraClean Microbial Kit (QIAGEN, Venlo, the Netherlands) according to manufacturer's instructions, followed by quantification of their concentration through qPCR with a QuantStudio ${ }^{\text {ma }}$ 5 Real-Time PCR System (Applied Biosystems, Foster City, CA, USA) using primers specific for L. rhamnosus GG (Brandt and Alatossava, 2003), S. cerevisiae (Zott et al., 2010) and ETEC (Taniuchi et al., 2012). The qPCR protocols of $L$. rhamnosus GG and S. cerevisiae were optimised in order to allow quantification of both strains in the presence of a complex background microbiota. The qPCR assays resulted in an efficiency of 77 and $85 \%$ for L. rhamnosus GG and S. cerevisiae respectively, with good reproducibility between biological replicated. The mucin agar of the mucus beads was harvested after $48 \mathrm{~h}$ of incubation. DNA extraction was performed on $0.25 \mathrm{~g}$ of mucus followed by specific qPCR to study the adhesion of the strains to the mucosal layer.

\section{ETEC toxin determination}

The concentration of heat-labile toxin produced by ETEC was determined after $0,6,24$, and $48 \mathrm{~h}$ of colonic incubation through GM1 ELISA as described previously by Salimian $e t$ al. (2010) with some minor modifications. Absorbance was measured using the Synergy HT Microplate reader (BioTek, Bad Friedrichshall, Germany). Both the concentration of toxin present in the fermentation liquid as well as the concentration of toxin that was bound to the cell wall were determined by separating the supernatant and cell pellet originating from $1 \mathrm{ml}$ of sample. The cell pellet was resuspended in PBS and sonicated prior to toxin quantification.

\section{Microbial metabolic activity}

Short-chain fatty acids (SCFA), lactate, ammonium and ethanol production were measured at the start of the incubation, and after $48 \mathrm{~h}$. Lactate quantification was performed using a commercially available enzymatic assay kit (R-Biopharm, Darmstadt, Germany) according to the manufacturer's instructions. Ammonium analysis was performed using a KjelMaster K-375 device (Büchi, Hendrik-Ido-Ambacht, the Netherlands). Ammonium in the sample was liberated as ammonia by the addition of 
$32 \% \mathrm{NaOH}$. The released ammonia was distilled from the sample into a $2 \%$ boric acid solution. Finally, the ammonium in the distillate was determined by titration with a $0.02 \mathrm{M}$ $\mathrm{HCl}$ solution. Short chain fatty acids (SCFA), including acetate, propionate, butyrate and branched SCFA (sum of isobutyrate, isovalerate and isocaproate), and ethanol were extracted by the addition of $0.1 \mathrm{~g}$ of $\mathrm{NaCl}$ and an acetonitrile mixture, containing $99.5 \%$ acetonitrile and $0.5 \%$ formic acid, in a 1:1 ratio. As an internal standard, 2-methyl hexanoic acid was added to all samples. Suspensions were centrifuged for $15 \mathrm{~min}$ at $15,080 \times \mathrm{g}$ after which concentrations of SCFA and ethanol were determined using a GC-2014 gas chromatograph with flame ionisation (FID) (Shimadzu, 's-Hertogenbosch, the Netherlands). Nitrogen was used as a carrier gas at a constant flow rate of $2.19 \mathrm{ml} / \mathrm{min}$ and as a make-up gas. The injector and detector temperatures were set at 200 and $240^{\circ} \mathrm{C}$, respectively. The column temperature program was as follows: $0 \mathrm{~min} 40^{\circ} \mathrm{C}$; $18 \mathrm{~min} 220^{\circ} \mathrm{C}$ and $23 \mathrm{~min} 220^{\circ} \mathrm{C}$. The injection volume was $1 \mu \mathrm{l}$ and a split ratio of 40 was applied.

\section{Agar well diffusion assay}

First, second-generation cultures of ETEC, L. rhamnosus GG, and S. boulardii were prepared in Luria-Bertani (LB) medium, De Man-Rogosa-Sharpe (MRS) medium, and yeast extract peptone dextrose (YEPD) medium, respectively, by growing them during each generation for $24 \mathrm{~h}$ at $37^{\circ} \mathrm{C}$ under normal atmospheric conditions. L. rhamnosus GG and S. boulardii cells were harvested and four aliquots were prepared: (1) $3 \mathrm{ml}$ of the second-generation culture was centrifuged for $5 \mathrm{~min}$ at $1,500 \times g$, the supernatant (pH of 4.4 for L. rhamnosus GG and 5.5 for S. boulardii) was collected and subsequently filtered (pore size of $0.2 \mu \mathrm{m}$ ) into a sterile recipient; (2) $3 \mathrm{ml}$ of the secondgeneration culture was centrifuged for $5 \mathrm{~min}$ at $1,500 \times \mathrm{g}$, the supernatant was collected and $\mathrm{pH}$ adjusted to 6.5 using $1 \mathrm{M} \mathrm{NaOH}$ or $1 \mathrm{M} \mathrm{HCl}$; (3) $3 \mathrm{ml}$ of the second-generation culture was sonicated for $2 \mathrm{~min}$; (4) $2 \mathrm{ml}$ of the secondgeneration culture was centrifuged for $5 \mathrm{~min}$ at $5,000 \times g$, and the pellet was resuspended in an equal amount of PBS. ETEC was prepared by centrifuging $2 \mathrm{ml}$ of a secondgeneration culture for $5 \mathrm{~min}$ at $1,500 \times g$. The resulting cell pellet was resuspended in $6 \mathrm{ml}$ of sterile PBS to obtain an ETEC solution at $10^{8} \mathrm{cfu} / \mathrm{ml}$.

The agar well diffusion assay was performed by first adding $0.5 \mathrm{ml}$ of the resuspended ETEC culture to big petri dishes followed by the addition of $20 \mathrm{ml}$ of YEPD agar medium. The plates were swung to mix ETEC equally over the agar plate. After drying the plates, were subsequently dried. Afterwards holes were punched in the solidified agar using a sterile Pasteur pipet to create wells. The four test aliquots of L. rhamnosus GG and S. boulardii were mixed with an equal amount of $2 \times$ concentrated molten MRS agar medium and $2 \times$ concentrated molten YEPD agar medium, respectively. The wells in the YEPD agar plates seeded with ETEC, were filled with the molten agar media containing the test solutions. The plates were allowed to dry and were subsequently incubated for $24 \mathrm{~h}$ at $37{ }^{\circ} \mathrm{C}$ under normal atmospheric conditions.

\section{Statistics}

All experiments were performed in biological triplicate. Statistical analysis was performed using the SPSS Statistics software, version 25 (SPSS, Chicago, IL, USA). Normality of data and equality of the variances were determined with a Shapiro-Wilk test and a Levene's test, respectively. For normally distributed data with equal variances a one-way ANOVA with a Bonferroni post-hoc test was used. For normally distributed data with unequal variances, a Welch test with a Games-Howell post-hoc test was conducted. For non-normally distributed data, a Kruskal-Wallis one-way ANOVA test with multiple post-hoc pairwise comparisons were performed. Bonferroni corrections were implemented on the significance levels of the multiple pairwise comparisons. In terms of statistics, the differences for all data discussed and indicated by ' $P<0.05$ ' were significant with a confidence interval of $95 \%$.

\section{Results}

\section{Growth of Lactobacillus rhamnosus GG and Saccharomyces c. boulardii}

Growth of L. rhamnosus GG and S. boulardii was measured in the presence of a healthy colonic background microbiota of both an adult (Figure 1) and a toddler (Supplementary Figure S1) donor. Similar effects were observed for both donors. During control incubations, as well as during the incubations to which S. boulardii was added, low background levels of $L$. rhamnosus GG were detected throughout the incubation period. Addition of L. rhamnosus GG to the incubations (alone or in combination with $S$. boulardii) resulted in initial concentrations of $9.1 \log \mathrm{cfu} /$ $\mathrm{ml}$, followed by moderate increases up to a maximum concentration of $9.7 \log \mathrm{cfu} / \mathrm{ml}$ throughout the incubation period. On the contrary, inoculating $S$. boulardii to the incubations resulted in initial concentrations of $7.7 \log \mathrm{cfu} /$ $\mathrm{ml}$ when S. boulardii was inoculated alone, and 7.4 $\log \mathrm{cfu} /$ $\mathrm{ml}$ when $S$. boulardii was supplemented in combination with L. rhamnosus GG, followed by a reduction towards the end of the incubation period. In the adult model, significantly higher concentrations of $S$. boulardii were present after 24 and $48 \mathrm{~h}$ of incubation compared to the incubations that were performed with $S$. boulardii alone.

The possible adhesion of L. rhamnosus GG and S. boulardii to the mucosal layer was studied by the addition of mucincoated beads to the colon medium. Supplementation of $L$. rhamnosus GG resulted in the presence of $7.2 \log \mathrm{cfu} / \mathrm{ml}$ 

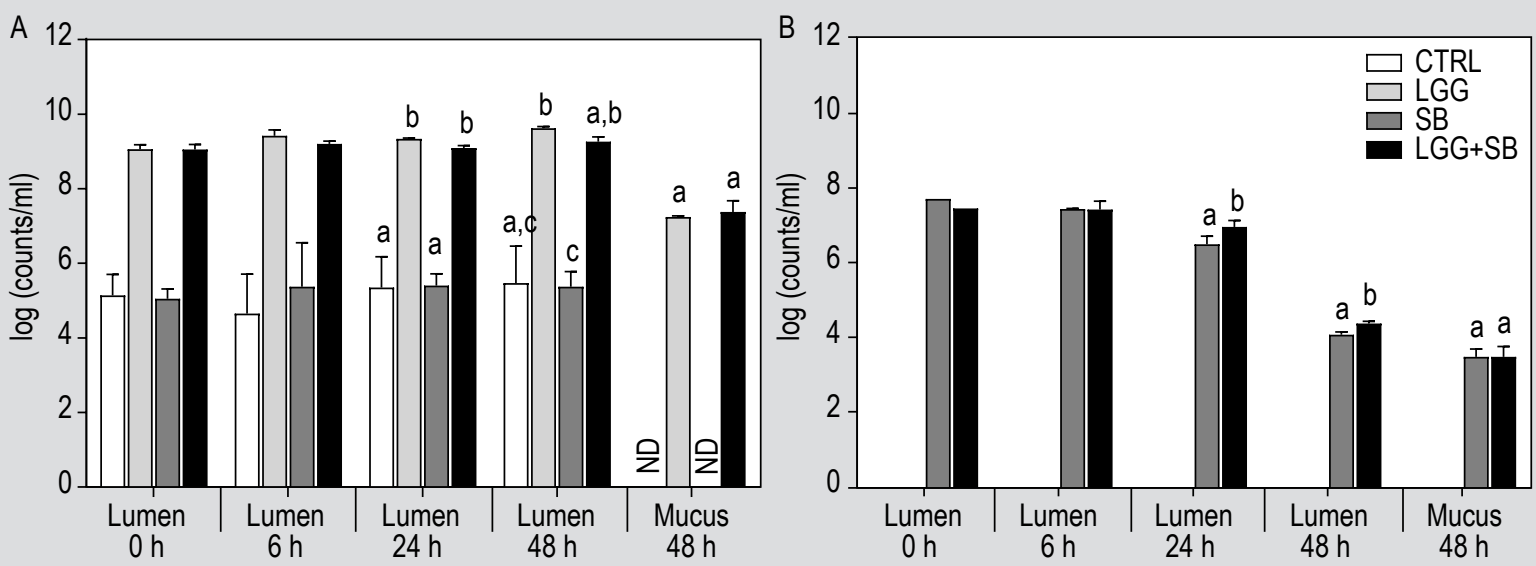

Figure 1. Growth of (A) Lactobacillus rhamnosus GG (LGG) and (B) Saccharomyces boulardii (SB) in microbiota colonic incubations from an adult donor. Mean log count $/ \mathrm{ml}$ ( \pm standard deviation) of $L G G$ and in the luminal and mucosal environment at several time points during the short-term colonic incubations using the colonic background microbiota of an adult donor $(n=3)$. LGG and SB were tested separately as well as together (LGG+SB) compared to a control incubation (CTRL). Statistically significant differences between experimental conditions (i.e. CTRL, LGG, SB and LGG+SB) after 24 and $48 \mathrm{~h}$ of incubation are highlighted by assigning different letters $(P<0.05)$. ND $=$ not detectable.

for the adult donor and $6.8 \mathrm{log} \mathrm{cfu} / \mathrm{ml}$ for the toddler donor after $48 \mathrm{~h}$ of incubation. Mucosal binding of S. boulardii was lower compared to L. rhamnosus GG, reaching concentrations of $3.5 \log \mathrm{cfu} / \mathrm{ml}$ and $3.8 \log \mathrm{cfu} / \mathrm{ml}$ for the adult and toddler donor, respectively. When $L$. rhamnosus GG and S. boulardii were supplemented together, a similar adhesion of the mucosal layer was observed.

\section{Microbial activity of Lactobacillus rhamnosus GG and Saccharomyces $c$. boulardii under healthy and dysbiotic conditions}

During the control incubations with the adult donor, an overall SCFA production of $86.5 \mathrm{mM}$, mainly attributed to acetate, propionate and butyrate, was observed under healthy conditions (Table 1), which indicated the high metabolic activity of the donor microbiota. When $L$. rhamnosus GG was added to the incubations, acetate levels significantly decreased compared to the control incubations. This coincided with statistically significant higher levels of butyrate during these incubations, i.e. 25.0 $\mathrm{mM}$ compared to $17.0 \mathrm{mM}$ for the control incubations. During the incubations with $S$. boulardii significantly more propionate was produced compared to the control experiments. Administration of both L. rhamnosus GG and $S$. boulardii to the colonic microbiota resulted in higher concentrations of both butyrate and propionate compared to the control experiments, indicating the additive activity of both strains on the colonic microbiota.

Under dysbiotic conditions (Table 2), SCFA production was significantly lower during the control incubations compared to the healthy conditions $(P=0.008$ for acetate,
$P=0.009$ for propionate and $P<0.001$ for butyrate), with the strongest reduction being observed for butyrate production, i.e. a reduction of $13.5 \mathrm{mM}$. Addition of $L$. rhamnosus GG, S. boulardii, or both had no major effect on SCFA production under dysbiotic conditions. Overall, similar trends in SCFA production were observed during the experiments performed with the toddler donor (Supplementary Table S1 for healthy conditions and Table S2 for dysbiotic conditions), albeit not reaching statistical

Table 1. Overall metabolic activity under simulated colonic healthy conditions for adult donor. ${ }^{1,2}$

\begin{tabular}{lllll} 
& CTRL & LGG & SB & LGG + SB \\
Acetate $(\mathrm{mM})$ & $51.9^{\mathrm{a}} \pm 0.7$ & $39.7^{\mathrm{b}} \pm 2.1$ & $49.2^{\mathrm{c}} \pm 0.3$ & $46.1^{\mathrm{b}} \pm 0.7$ \\
Propionate $(\mathrm{mM})$ & $17.6^{\mathrm{a}} \pm 0.4$ & $14.8^{\mathrm{b}} \pm 0.8$ & $19.7^{\mathrm{c}} \pm 0.3$ & $19.2^{\mathrm{c}} \pm 0.4$ \\
Butyrate $(\mathrm{mM})$ & $17.0^{\mathrm{a}} \pm 0.4$ & $25.0^{\mathrm{b}} \pm 0.2$ & $12.4^{\mathrm{c}} \pm 0.1$ & $17.7^{\mathrm{d}} \pm 0.2$ \\
Lactate $(\mathrm{mM})$ & $0.5^{\mathrm{a}} \pm 0.2$ & $0.4^{\mathrm{a}} \pm 0.1$ & $2.1^{\mathrm{a}} \mathrm{b}^{\mathrm{b}} \pm 0.6$ & $1.3^{\mathrm{b}} \pm 0.2$ \\
Ethanol (mM) & $8.6^{\mathrm{a}} \pm 1.0$ & $7.5^{\mathrm{a}} \pm 1.1$ & $29.2^{\mathrm{b}} \pm 0.7$ & $20.0^{\mathrm{c}} \pm 1.2$ \\
Branched SCFA (mM) & $1.4^{\mathrm{a}} \pm 0.0$ & $1.5^{\mathrm{a}} \pm 0.0$ & $4.3^{\mathrm{b}} \pm 0.1$ & $4.5^{\mathrm{c}} \pm 0.1$ \\
Ammonium (mg/l) & $317.9^{\mathrm{a}} \pm 16.3$ & $328.2^{\mathrm{a}} \pm 19.7$ & $437.5^{\mathrm{b}} \pm 9.6$ & $426.3^{\mathrm{b}} \pm 29.5$ \\
\hline
\end{tabular}

${ }^{1}$ Average change ( \pm standard deviation) in acetate, propionate, butyrate, lactate, ethano, branched short chain fatty acids (SCFA) and ammonium between $0-48 \mathrm{~h}$ during the short-term colonic incubations using a colonic background microbiota of an adult donor $(n=3)$.

${ }^{2}$ Lactobacillus rhamnosus GG (LGG) and Saccharomyces boulardii (SB) were tested separately as well as together (LGG+SB) compared to a control incubation (CTRL). Statistically significant differences between experimental conditions (i.e. CTRL, LGG, SB and LGG+SB) after $48 \mathrm{~h}$ of incubation are highlighted by assigning different letters $(P<0.05)$. 
Table 2. Overall metabolic activity under simulated colonic dysbiotic conditions for adult donor. ${ }^{1,2}$

\begin{tabular}{lllll} 
& CTRL & LGG & SB & LGG + SB \\
Acetate $(\mathrm{mM})$ & $42.7^{\mathrm{a}} \pm 3.2$ & $44.3^{\mathrm{a}} \pm 3.3$ & $45.5^{\mathrm{a}} \pm 7.8$ & $42.3^{\mathrm{a}} \pm 1.8$ \\
Propionate $(\mathrm{mM})$ & $13.3^{\mathrm{a}, \mathrm{b}} \pm 1.5$ & $10.4^{\mathrm{a}} \pm 1.0$ & $13.8^{\mathrm{a}, \mathrm{b}} \pm 1.6$ & $13.8^{\mathrm{b}} \pm 0.6$ \\
Butyrate $(\mathrm{mM})$ & $3.5^{\mathrm{a}} \pm 0.6$ & $6.2^{\mathrm{a}} \pm 0.4$ & $3.8^{\mathrm{a}} \pm 0.3$ & $5.5^{\mathrm{a}} \pm 0.2$ \\
Lactate $(\mathrm{mM})$ & $-0.2^{\mathrm{a}} \pm 0.0$ & $0.0^{\mathrm{b}} \pm 0.0$ & $-0.1^{\mathrm{c}} \pm 0.0$ & $-0.1^{\mathrm{d}} \pm 0.0$ \\
Ethanol $(\mathrm{mM})$ & $11.0^{\mathrm{a}} \pm 1.0$ & $10.8^{\mathrm{a}} \pm 0.7$ & $17.9^{\mathrm{b}} \pm 2.2$ & $14.1^{\mathrm{a}} \pm 0.3$ \\
Branched SCFA (mM) & $1.4^{\mathrm{a}} \pm 0.6^{\mathrm{a}} \pm$ & $1.0^{\mathrm{a}} \pm 0.0$ & $1.7^{\mathrm{a}} \pm 0.1$ & $1.3^{\mathrm{a}} \pm 0.1$ \\
Ammonium (mg/l) & $265.3^{\mathrm{a}} \pm 3.4$ & $259.1^{\mathrm{a}, \mathrm{b}} \pm 14.4$ & $276.1^{\mathrm{a}, \mathrm{b}} \pm 19.6$ & $288.9^{\mathrm{b}} \pm 2.1$ \\
\hline
\end{tabular}

${ }^{1}$ Average change ( \pm standard deviation) in acetate, propionate, butyrate, lactate, ethanol, branched short chain fatty acids (SCFA) and ammonium between $0-48 \mathrm{~h}$ during the short-term colonic incubations using an adult colonic background microbiota supplemented with Escherichia coli ETEC $(n=3)$.

2 Lactobacillus rhamnosus GG (LGG) and Saccharomyces boulardii (SB) were tested separately as well as together ( $(\mathrm{GG}+\mathrm{SB})$ compared to a control incubation (CTRL). Statistically significant differences between experimental conditions (i.e. CTRL, LGG, SB and LGG+SB) after $48 \mathrm{~h}$ of incubation are highlighted by assigning different letters $(P<0.05)$.

significance except for a significant increase in butyrate levels upon supplementation of LGG and LGG+SB under healthy conditions.

When comparing healthy and dysbiotic conditions for the control incubations, it was observed that in the adult model lactate levels were significantly higher under healthy conditions $(P=0.046)$, while in the toddler model a trend towards higher lactate levels was observed under healthy conditions $(P=0.060)$. Upon addition of $L$. rhamnosus $\mathrm{GG}$, lactate levels remained unaffected compared to the control incubations, except for a significant increase for the adult donor under dysbiotic conditions (Table 2). Addition of S. boulardii to the colonic incubations of the adult donor resulted in increased lactate concentrations compared to the control incubations under both healthy (Table 1) and dysbiotic (Table 2) conditions, whereas decreased lactate levels were observed for the toddler donor (Supplementary Table S1 and S2), though only reaching significance under dysbiotic conditions. Dosing of L. rhamnosus GG together with $S$. boulardii resulted in an averaged outcome of the effects they both generated when incubated alone.

Ethanol was produced during the control experiments with both donors under healthy (Table 1 for adult and Supplementary Table S1 for toddler) and dysbiotic (Table 2 for adult and Supplementary Table S2 for toddler) conditions. Under dysbiotic conditions, ethanol concentrations were higher compared to healthy conditions for the control incubations, albeit only reaching significance in the adult model $(P=0.042)$. Ethanol levels remained unaffected upon dosing of L. rhamnosus GG to the colonic incubations. Addition of S. boulardii resulted in a statistically significant increase in the production of ethanol compared to the control experiments in the adult model, whereas for the toddler donor under healthy conditions, a trend towards increased ethanol concentrations was observed. When S. boulardii and L. rhamnosus GG were administered together to the adult model, significantly increased ethanol production was observed under healthy conditions compared to the control experiments, though still significantly lower than the increase observed when $S$. boulardii was inoculated alone.

Ammonium and branched chain fatty acids (BCFA) were produced during all experiments of the present study, indicating the occurrence of proteolytic fermentation. When comparing healthy and dysbiotic conditions for the control incubations, it was observed that ammonium and BCFA levels were significantly higher under healthy conditions in both donors tested, except for BCFA levels in the adult model where similar levels were observed between both conditions. Addition of L. rhamnosus GG to the colonic incubations did not result in increased production of ammonium and BCFA compared to the control incubations under both healthy (Table 1 for adult and Supplementary Table S1 for toddler) and dysbiotic (Table 2 for adult and Supplementary Table S2 for toddler) conditions. Under healthy conditions, supplementation of $S$. boulardii (alone or in combination with L. rhamnosus GG) resulted in a statistically significant increase in ammonium and BCFA production compared to the control experiments, for both donors tested. Under dysbiotic conditions, supplementation of $L$. rhamnosus GG together with S. boulardii resulted in statistically significantly higher ammonium production compared to the control experiments when using an adult $(P<0.004)$ and a toddler $(P=0.050)$ colonic background microbiota, whereas no statistically significant differences were observed for BCFA production.

\section{Effect of Lactobacillus rhamnosus GG and Saccharomyces c. boulardii against ETEC}

Determination of the growth of ETEC during the control incubations demonstrated that this pathogenic strain was capable to outgrow in the presence of the dysbiotic colonic background community of both the adult (Figure 2) and toddler (Supplementary Figure S2) donor. Indeed, during both sets of experiments, ETEC reached high cell abundances after $24 \mathrm{~h}$ of incubation. Administration of $S$. boulardii to the experiments had no major effect on the growth of ETEC. On the contrary, supplementation of $L$. rhamnosus GG resulted in lower concentrations of ETEC throughout the course of the experiments, with the strongest effect being observed after $24 \mathrm{~h}$, when both L. rhamnosus GG and S. boulardii were added to the 


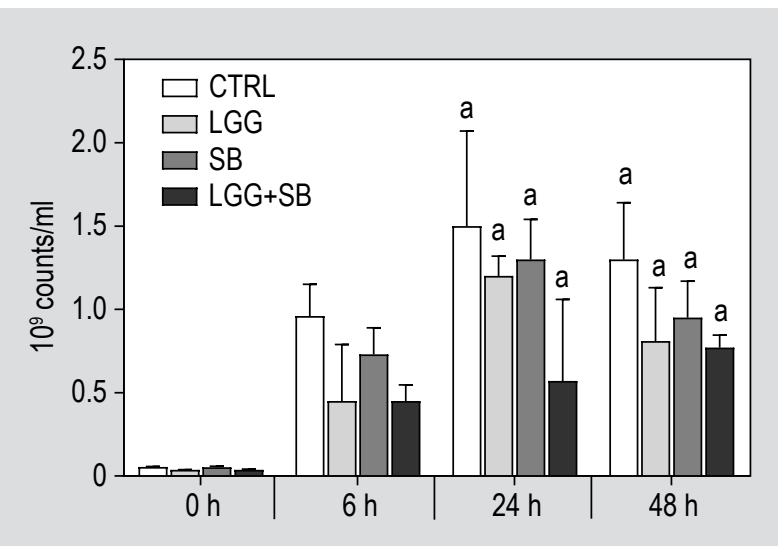

Figure 2. Growth of Escherichia coli ETEC in microbiota colonic incubations from an adult donor. Mean counts/ml $( \pm$ standard deviation) of ETEC in the lumen at several time points during the short-term colonic incubations using a colonic background microbiota of an adult donor supplemented with ETEC $(n=3)$. Lactobacillus rhamnosus GG (LGG) and Saccharomyces boulardii (SB) were tested separately as well as together (LGG+SB) compared to a control incubation (CTRL). Statistically significant differences between experimental conditions (i.e. CTRL, LGG, SB and LGG+SB) after 24 and $48 \mathrm{~h}$ of incubation are highlighted by assigning different letters $(P<0.05)$.

incubations. A 40 and $46 \%$ reduction in the concentration of ETEC was observed upon addition of both strains during the experiments with the adult donor and toddler donor, respectively, reaching statistical significance during the experiments using the toddler colonic background community $(P=0.013)$.

ETEC was able to adhere to the mucus beads for both the adult (Figure 3) and the toddler (Supplementary Figure S3) donor, resulting in an average adhesion of 6.6 and 7.0 $\log \mathrm{cfu} / \mathrm{g}$, respectively. L. rhamnosus GG and S. boulardii as well as their combination did not reduce ETEC binding for both donors.

The results obtained during the agar well diffusion experiments (Table 3) indicated that the culture supernatant of $L$. rhamnosus GG had an antimicrobial effect on ETEC. This was mainly due to the low $\mathrm{pH}$ of the supernatant $(\mathrm{pH}=4.4)$ since no inhibition zones were obtained when the culture supernatant was adjusted to $\mathrm{pH}$ 6.5. Large inhibition zones were obtained when the sonicated cell suspensions of $L$. rhamnosus GG were administered to the plates. Finally, metabolically active cells of $L$. rhamnosus GG also resulted in the occurrence of inhibition zones. The culture supernatant of $S$. boulardii had no antimicrobial effect against ETEC. On the contrary, the sonicated fraction of the S. boulardii culture resulted in inhibition zones through its antimicrobial activity against ETEC. Finally, also the metabolically active $S$. boulardii cells resulted in inhibition zones.

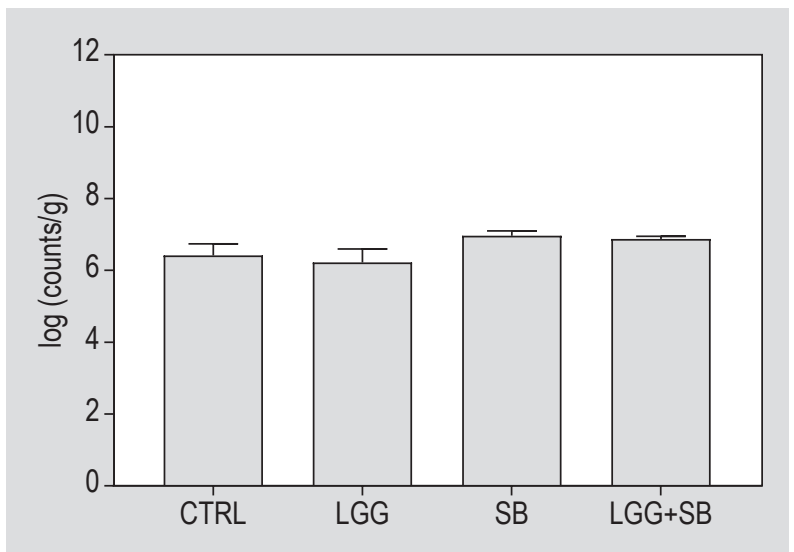

Figure 3. Growth of mucosal Escherichia coli ETEC in microbiota colonic incubations from an adult donor. Mean counts $/ \mathrm{ml}( \pm$ standard deviation) of ETEC in the mucus after $48 \mathrm{~h}$ during the short-term colonic incubations using a colonic background microbiota of an adult donor supplemented with ETEC $(n=3)$. Lactobacillus rhamnosus GG (LGG) and Saccharomyces boulardii (SB) were tested separately as well as together ( $L G G+S B$ ) compared to a control incubation (CTRL). Statistically significant differences between experimental conditions (i.e. CTRL, LGG, SB and LGG+SB) after $48 \mathrm{~h}$ of incubation are highlighted by assigning different letters $(P<0.05)$.

Determination of the concentration of toxin produced in the supernatant revealed that the growth of ETEC resulted in the production of its toxin over the course of the control incubations and this for both donors (Figure 4 for adult and Supplementary Figure S5 for toddler). Administration of L. rhamnosus GG to the incubations had no effect on the production of the toxin by ETEC in the supernatant. On the

Table 3. Agar well diffusion assay. ${ }^{1}$

\begin{tabular}{lll} 
Probiotic & Fraction & Agar well diffusion ${ }^{2}$ \\
Lactobacillus & Supernatant & $2 \mathrm{~mm}$ inhibition zone \\
rhamnosus GG & Supernatant; neutral pH & No inhibition zone \\
& Sonicated & $4 \mathrm{~mm}$ inhibition zone \\
& Live cells & $3 \mathrm{~mm}$ inhibition zone \\
& Succharomyces \\
boulardii & Supernatant & No inhibition zone \\
& Sonicated & No inhibition zone \\
& Live cells & $2 \mathrm{~mm}$ inhibition zone ${ }^{2}$ \\
& & $1 \mathrm{~mm}$ inhibition zone ${ }^{2}$ \\
\hline
\end{tabular}

${ }^{1}$ Antimicrobial activity of different Lactobacillus rhamnosus GG and Saccharomyces boulardii fractions (i.e. supernatant, supernatant at neutral $\mathrm{pH}$, sonicated cells and live cells) against Escherichia coli ETEC as determined by measuring the inhibition zone in the agar well diffusion assay.

${ }^{2}$ Shown in Supplementary Figure S4. 

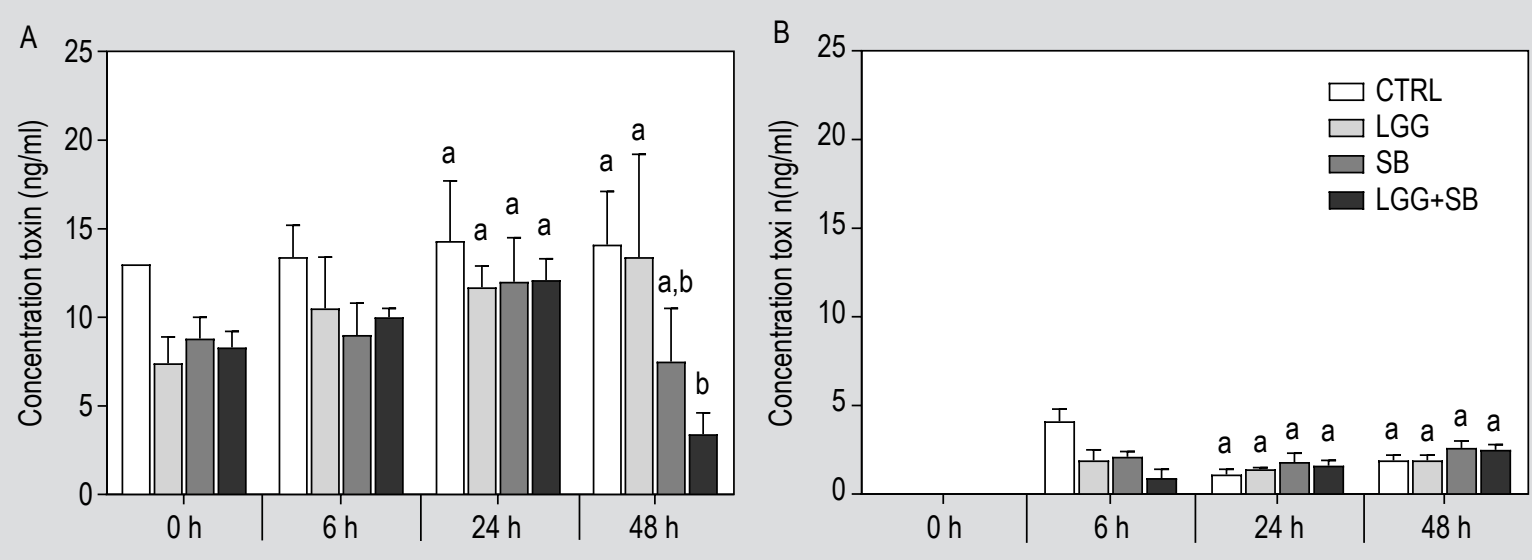

Figure 4. Escherichia coli ETEC toxins in a colonic microbiota of an adult donor. Average concentration (ng/ml) $( \pm$ standard deviation) of ETEC toxin present in (A) the supernatant and (B) bound to the bacterial cells (pellet) at several time points during the short-term colonic incubations using a colonic background microbiota of an adult donor supplemented with ETEC $(n=3)$. Lactobacillus rhamnosus GG (LGG) and Saccharomyces boulardii (SB) were tested separately as well as together (LGG+SB) compared to a control incubation (CTRL). Statistically significant differences between experimental conditions (i.e. CTRL, LGG, SB and LGG+SB) after 24 and $48 \mathrm{~h}$ of incubation are highlighted by assigning different letters $(P<0.05)$.

contrary, lower concentrations of toxin were present in the supernatant after $48 \mathrm{~h}$ of incubation during the experiments with $S$. boulardii, albeit not statistically significant, and this for both donors. During the experiments to which both L. rhamnosus GG and S. boulardii were supplemented, ETEC was capable to produce its toxin during the first 6 and 24 h of incubation resulting in elevated toxin levels in the supernatant. The concentration of toxin subsequently decreased between 24 and $48 \mathrm{~h}$ of incubation to a significantly lower concentration compared to the control incubations and this both for the adult $(P=0.015)$ and toddler $(P=0.006)$ donor. The concentration of cell wallbound toxin was lower compared to the concentration in the supernatant and this for all different sets of experiments. Administration of L. rhamnosus GG, S. boulardii, or L. rhamnosus GG together with $S$. boulardii had no effect on the concentration of toxin that was bound to the cell wall. The sum of the concentrations of the toxin in the supernatant and bound to the cell wall showed that $L$. rhamnosus GG and S. boulardii had a synergistic effect against toxin production by ETEC and this both for the adult donor $(P=0.018)$ and for the toddler $(P=0.055)$ donor, resulting in a reduction of 57.4 and $45.5 \%$, respectively.

\section{Discussion}

To our knowledge, the well-documented strains $L$. rhamnosus GG and S. boulardii have never been studied together before. As both strains confer several health benefits, we aimed to study the interaction between $L$. rhamnosus GG and S. boulardii at concentrations which are in the range of usual therapeutic doses in humans, their modulatory effect on the colonic microbial functionality and the possible synergistic effects when both strains are added together into a healthy colonic environment using an in vitro model. Two different donors were used as a source of a colonic background community, and this to account for inter-individual differences. As the microbiome in toddlers is similar to that in adults (Voreades et al., 2014), both types of donors were selected for the current study.

Addition of L. rhamnosus GG to the colonic incubations resulted in a high concentration of this strain at the start of the experiments, followed by minor growth throughout the incubation period. Furthermore, determination of $L$. rhamnosus GG concentrations present on the mucin-coated beads revealed that this strain was capable to adhere to the mucosal layer of the simulated colon. Indeed, Kankainen et al. (2009) revealed that the surface of L. rhamnosus GG contains mucus-binding pili, which shows its ability to bind to the mucosal layer in the colon. Co-cultivation of L. rhamnosus GG together with $S$. boulardii resulted in comparable concentrations of $L$. rhamnosus GG in both the luminal and mucosal compartments of the incubations, which indicated that the activity of $S$. boulardii had no negative impact on L. rhamnosus GG in the lumen and that S. boulardii did not compete with L. rhamnosus GG for adhesion sites in the mucosa. Dosing of $S$. boulardii to the incubations resulted in high concentrations of this strain during the first $6 \mathrm{~h}$ of the experiments. Afterwards, levels of $S$. boulardii decreased and this especially after $48 \mathrm{~h}$. This was probably due to competition for substrates with the colonic background microbiota and not due to competition with L. rhamnosus GG, as this occurred when S. boulardii was grown alone and similar effects were observed when both strains were added to the incubations. In the adult model, even significantly higher concentrations of $S$. boulardii were observed after 24 and 48 h of incubation 
compared to the incubations that were performed with $S$. boulardii alone. This could indicate a protective effect of L. rhamnosus GG towards $S$. boulardii. Furthermore, $S$. boulardii was also capable of binding the mucin-coated beads present in the incubations and its adhesion was not affected by the presence of $L$. rhamnosus GG. Adhesion was lower compared with L. rhamnosus GG, which could be explained by the absence of mucus-binding pili for $S$. boulardii (Moré and Swidsinski, 2015).

Determination of the metabolites produced during these experiments revealed the additive effect of L. rhamnosus GG and S. boulardii when inoculated together to the colon under healthy conditions. Dosing of L. rhamnosus GG to the colonic microbiota resulted in the production of increased levels of butyrate compared to the control. Butyrate is a major energy source for colonocytes (Basson et al., 1996) and has been associated with several healthpromoting properties, such as reduction of oxidative stress, improvement of gut barrier function and enhancement of anti-inflammatory response (Hamer et al., 2008). As one of the main end metabolites of L. rhamnosus GG fermentation is lactate (De Keersmaecker et al., 2006), the observed increase in butyrate levels was probably attributed to stimulation of specific lactate-utilising, butyrate-producing bacterial species such as Anaerostipes caccae and Eubacterium hallii (Duncan et al., 2004). The utilisation of lactate by these microorganisms probably resulted in an absence of increase on lactate levels during the incubation period due to production and subsequent consumption of this transient metabolite (Moens et al., 2017). Furthermore, during conversion of lactate into butyrate, acetate is an essential co-substrate that needs to be consumed to complete the final reaction step in butyrate synthesis (Duncan et al., 2002), which was indeed observed by a reduction of acetate levels upon dosing $L$. rhamnosus GG to the experiments. Dosing of S. boulardii gave rise to the production of high concentrations of ethanol. Ethanol is known for its antimicrobial properties but is in general not a major end-metabolite produced during colonic fermentation processes. Therefore, ethanol production can mainly be attributed to the fermentative activity of $S$. boulardii during the aforementioned incubations. Furthermore, addition of S. boulardii to the colonic microbiota resulted in increased production of propionate throughout the course of the incubation, which could be linked to the decreased concentrations of luminal S. boulardii after 48 h of incubation. Indeed, the decreased $S$. boulardii concentrations suggested the occurrence of cell lysis resulting in the release of major cell wall components in the medium, which could be used as substrates by propionate-producing bacteria. Cuskin et al. (2015) reported that the propionate-producing Bacteroidetes species can utilise mannan-oligosaccharides, which are present in the outer cell wall of yeast. Hughes et al. (2008) reported that beta-glucans, another major cell wall component of yeasts (Manners et al., 1973), specifically stimulate propionate during colonic fermentation. Cocultivation of both L. rhamnosus GG and S. boulardii resulted in increased butyrate, propionate, and ethanol production compared to the control incubations indicating the cooperative effect of both strains.

Moreover, the antipathogenic effect of $L$. rhamnosus GG and $S$. boulardii against ETEC was investigated in a colonic dysbiotic environment. Dysbiosis was confirmed by the significantly decreased levels of SCFA and ammonium compared to healthy conditions. ETEC was capable to outgrow in the dysbiotic microbiota of both donors and to adhere to the mucosal surface. Addition of L. rhamnosus GG to the incubations resulted in lower concentrations of luminal ETEC at each sampling point, with the lowest concentration of ETEC observed when L. rhamnosus GG and $S$. boulardii were dosed together. The antimicrobial activity of $L$. rhamnosus GG was mainly attributed to the active production of toxic metabolites as large inhibition zones were obtained during the agar well diffusion assay in the presence of the culture supernatant at low environmental $\mathrm{pH}$. The antimicrobial activity of L. rhamnosus GG was probably attributed to the production of lactate, as was already reported by De Keersmaecker et al. (2006). Lactate decreases the $\mathrm{pH}$ of the environment (Macfarlane and Englyst, 1986). Especially at low $\mathrm{pH}$ values, lactate can exert strong antimicrobial effects against pathogens, as protonated lactic acid can penetrate the microbial cell after which it dissociates and releases protons within the cell, resulting in acidification and microbial cell death (Alakomi et al., 2000; Raybaudi-Massilia et al., 2009; Stratford and Eklund, 2003). Therefore, the antimicrobial activity of $L$. rhamnosus GG could be attributed to lactate production under acidic environmental conditions. Administration of $S$. boulardii also resulted in antimicrobial activity against ETEC in the luminal environment. The sonicated fraction of the S. boulardii culture resulted in the largest inhibition zones, indicating that cell components of S. boulardii were toxic for ETEC. Additionally, dosing of $S$. boulardii to the experiments resulted in lower toxin concentrations after $48 \mathrm{~h}$ of incubation compared to the control experiments, with an increased effect being observed when $L$. rhamnosus GG and S. boulardii were co-cultivated. The effect on toxin production by $S$. boulardii could be explained by the secretion of proteolytic enzymes. Indeed, the production of a specific protease by $S$. boulardii has already been shown to inhibit the effects of Clostridium difficile toxins A and B in human colonic mucosa (Castagliuolo et al., 1999). Also, Buts et al. (2006) reported that S. boulardii produces a protein phosphatase that inhibited $E$. coli endotoxins in the small intestine of rats. Furthermore, Roussel et al. (2018) demonstrated that the probiotic yeast Saccharomyces cerevisiae CNCM I-3856 reduces ETEC toxin concentrations in culture media through active removal of the toxin by the yeast strain. The increased 
ammonium and branched SCFA concentrations observed during the incubations with $S$. boulardii confirms the stimulation of proteolytic activity. Hence, addition of $L$. rhamnosus GG together with $S$. boulardii resulted in a stronger effect which was probably due to the fact that the growth of L. rhamnosus GG resulted in a faster and more pronounced depletion of carbohydrates thereby increasing the proteolytic activity of $S$. boulardii towards the toxin. This also explains why the toxin concentration initially increased during the experiments with $S$. boulardii to comparable concentrations as the control, as during the initial $24 \mathrm{~h}$ of the incubation carbohydrates were still present in the medium which stimulated saccharolytic fermentation by $S$. boulardii thereby minimising its proteolytic activity. While all antimicrobial activity against ETEC was observed in the luminal environment, both L. rhamnosus GG and S. boulardii were not capable to compete for binding sites with ETEC on the mucosa. However, the mucus layer generally creates a protective barrier (Cornick et al., 2015) and therefore one single dose of the test strains in the current experimental setup was probably not effective to observe antipathogenic effects in the mucosal environment. Whereas, L. rhamnosus GG and S. boulardii had a detrimental effect on ETEC in the lumen, they did not seem to exert these effects on the residing microbial community of both donors, as was seen by the stable SCFA levels compared to the control under these dysbiotic conditions. The activity of the strains even resulted in the production of additional butyrate (by $L$. rhamnosus GG) and ethanol (by S. boulardii) during the colonic incubations. Altogether, these results demonstrated the complementarity of the antipathogenic effects of these strains on ETEC, resulting in a significantly lower amount of ETEC toxin after $48 \mathrm{~h}$.

Some potential shortcomings of the study design include the absence of analysis of the microbial community composition. Indeed, in the current study, impact of L. rhamnosus GG and $S$. boulardii on the residing microbiota under both healthy and dysbiotic conditions was mainly investigated through assessment of microbial functionality. Analysis of microbial community composition might have provided further insight into the effect of both probiotic strains on the residing microbial community. Indeed, presence of specific microbial groups in the microbial community of the respective donors might have contributed to the observed synergistic effects and therefore investigation of the potential correlations between the observed effects and the initial microbial community composition might provide insight into the responsiveness of subjects to the probiotic formulation. Furthermore, the effect of both strains was examined upon administration of one single dose, while in vivo probiotics are generally consumed over several days or weeks. Inclusion of the long-term evaluation of repeated probiotic intake might have provided further insight on the effect of L. rhamnosus GG, S. boulardii and their combination against ETEC. Finally, in the current experimental setting, a dysbiotic microbial community was simulated by the administration of very low percentage of the background microbial community of the donors under investigation. The strength of this approach lies in the fact that the impact of inter-individual variation was minimised allowing for physiologically relevant comparisons between both healthy and impaired conditions. However, while dilution of the faecal inoculum will have resulted in a dysbiotic microbial community in a quantitative way, the qualitative microbiota composition might not be as accurate as would be observed in vivo.

In conclusion, the results indicated that L. rhamnosus GG and $S$. boulardii were capable to grow together and no antagonistic effects were observed between both strains. Co-cultivation showed or resulted in the complementary of both strains at the metabolic level, which potentiated the antipathogenic and antitoxin activity against ETEC. Altogether, the results reinforce the hypothesis that both probiotics together may help microbiota functionality, either in adults or toddlers and under healthy or impaired conditions. This effect could be of great interest under dysbiotic conditions such as during antibiotic therapy, when the colonic microbiota is more sensitive to invasion of pathogens. Future research is warranted to further explore this hypothesis.

\section{Supplementary material}

Supplementary material can be found online at https://doi. org/10.3920/BM2019.0064.

Figure S1. Growth of Lactobacillus rhamnosus GG and Saccharomyces boulardii in microbiota colonic incubations from a toddler donor.

Figure S2. Growth of Escherichia coli ETEC in microbiota colonic incubations from a toddler donor.

Figure S3. Growth of mucosal Escherichia coli ETEC in microbiota colonic incubations from a toddler donor.

Figure S4. Agar well diffusion assay.

Figure S5. Escherichia coli ETEC toxins in a colonic microbiota of a toddler donor.

Table S1. Overall metabolic activity under simulated colonic healthy conditions for toddler donor.

Table S2. Overall metabolic activity under simulated colonic dysbiosed conditions for toddler donor. 


\section{Acknowledgements}

This work was sponsored by Ipsen. The authors thank the donors who kindly supplied a faecal sample for the purpose of the study. Furthermore, we thank Dr Annie Tremblay and Dr Thomas Tompkins for critically reviewing the manuscript.

\section{Conflict of interest}

Mireia Morera is an employee of Ipsen Pharma SAS.

\section{References}

Alakomi, H.L., Skyttä, E., Saarela, M., Mattila-Sandholm, T., LatvaKala, K. and Helander, I.M., 2000. Lactic acid permeabilizes gramnegative bacteria by disrupting the outer membrane. Applied and Environmental Microbiology 66: 2001-2005.

Anonymous, 2006. Future directions for research on enterotoxigenic Escherichia coli vaccines for developing countries. Weekly Epidemiological Record 81: 97-104.

Badia, R., Zanello, G., Chevaleyre, C., Lizardo, R., Meurens, F., Martínez, P., Brufau, J. and Salmon, H., 2012. Effect of Saccharomyces cerevisiae var. boulardii and $\beta$-galactomannan oligosaccharide on porcine intestinal epithelial and dendritic cells challenged in vitro with Escherichia coli F4 (K88). Veterinary Research 43: 4.

Basson, M.D., Turowski, G.A., Rashid, Z., Hong, F. and Madri, J.A., 1996. Regulation of human colonic cell line proliferation and phenotype by sodium butyrate. Digestive Diseases and Sciences 41: 1986-1993.

Bin, P., Tang, Z., Liu, S., Chen, S., Xia, Y., Liu, J., Wu, H. and Zhu, G., 2018. Intestinal microbiota mediates Enterotoxigenic Escherichia coli-induced diarrhea in piglets. BMC Veterinary Research 14: 385.

Brandt, K. and Alatossava, T., 2003. Specific identification of certain probiotic Lactobacillus rhamnosus strains with PCR primers based on phage-related sequences. International Journal of Food Microbiology 84: 189-196.

Buts, J.P., Dekeyser, N., Stilmant, C., Delem, E., Smets, F. and Sokal, E., 2006. Saccharomyces boulardii produces in rat small intestine a novel protein phosphatase that inhibits Escherichia coli endotoxin by dephosphorylation. Pediatric Research 60: 24-29.

Castagliuolo, I., Riegler, M.F., Valenick, L., LaMont, J.T. and Pothoulakis, C., 1999. Saccharomyces boulardii protease inhibits the effects of Clostridium difficile toxins A and B in human colonic mucosa. Infection and Immunity 67: 302-307.

Cornick, S., Tawiah, A. and Chadee, K., 2015. Roles and regulation of the mucus barrier in the gut. Tissue Barriers 3: e982426-e982426.

Cuskin, F., Lowe, E.C., Temple, M.J., Zhu, Y., Cameron, E.A., Pudlo, N.A., Porter, N.T., Urs, K., Thompson, A.J., Cartmell, A., Rogowski, A., Hamilton, B.S., Chen, R., Tolbert, T.J., Piens, K., Bracke, D., Vervecken, W., Hakki, Z., Speciale, G., Munōz-Munōz, J.L., Day, A., Peña, M.J., McLean, R., Suits, M.D., Boraston, A.B., Atherly, T., Ziemer, C.J., Williams, S.J., Davies, G.J., Abbott, D.W., Martens, E.C. and Gilbert, H.J., 2015. Human gut bacteroidetes can utilize yeast mannan through a selfish mechanism. Nature 517: 165.
Czerucka, D., Dahan, S., Mograbi, B., Rossi, B. and Rampal, P., 2000. Saccharomyces boulardii preserves the barrier function and modulates the signal transduction pathway induced in enteropathogenic Escherichia coli-infected T84 cells. Infection and Immunity 68: 5998-6004.

Czerucka, D., Piche, T. and Rampal, P., 2007. Yeast as probiotics - Saccharomyces boulardii. Alimentary Pharmacology and Therapeutics 26: 767-778.

Dahan, S., Dalmasso, G., Imbert, V., Peyron, J.-F., Rampal, P. and Czerucka, D., 2003. Saccharomyces boulardii interferes with enterohemorrhagic Escherichia coli-induced signaling pathways in T84 cells. Infection and Immunity 71: 766-773.

Dalmasso, G., Loubat, A., Dahan, S., Calle, G., Rampal, P. and Czerucka, D., 2006. Saccharomyces boulardii prevents TNF-alpha-induced apoptosis in EHEC-infected T84 cells. Research in Microbiology 157: 456-465.

Dalton, C.B., Mintz, E.D., Wells, J.G., Bopp, C.A. and Tauxe, R.V., 1999. Outbreaks of enterotoxigenic Escherichia coli infection in American adults: a clinical and epidemiologic profile. Epidemiology and Infection 123: 9-16.

De Keersmaecker, S.C., Verhoeven, T.L., Desair, J., Marchal, K., Vanderleyden, J. and Nagy, I., 2006. Strong antimicrobial activity of Lactobacillus rhamnosus GG against Salmonella typhimurium is due to accumulation of lactic acid. FEMS Microbiology Letters 259: 89-96.

Doron, S., Snydman, D.R. and Gorbach, S.L., 2005. Lactobacillus GG: bacteriology and clinical applications. Gastroenterology Clinics of North America 34: 483-498.

Duncan, S.H., Barcenilla, A., Stewart, C.S., Pryde, S.E. and Flint, H.J., 2002. Acetate utilization and butyryl coenzyme A (CoA):acetate-CoA transferase in butyrate-producing bacteria from the human large intestine. Applied and Environmental Microbiology 68: 5186-5190.

Duncan, S.H., Louis, P. and Flint, H.J., 2004. Lactate-utilizing bacteria, isolated from human feces, that produce butyrate as a major fermentation product. Applied and Environmental Microbiology 70: 5810.

Gaastra, W. and Svennerholm, A.M., 1996. Colonization factors of human enterotoxigenic Escherichia coli (ETEC). Trends in Microbiology 4: 444-452.

Guandalini, S., Pensabene, L., Zikri, M.A., Dias, J.A., Casali, L.G., Hoekstra, H., Kolacek, S., Massar, K., Micetic-Turk, D., Papadopoulou, A., de Sousa, J.S., Sandhu, B., Szajewska, H. and Weizman, Z., 2000. Lactobacillus GG administered in oral rehydration solution to children with acute diarrhea: a multicenter European trial. Journal of Pediatric Gastroenterology and Nutrition 30: $54-60$.

Hamer, H.M., Jonkers, D., Venema, K., Vanhoutvin, S., Troost, F.J. and Brummer, R.J., 2008. Review article: the role of butyrate on colonic function. Alimentary Pharmacology \& Therapeutics 27: 104-119. Hill, C., Guarner, F., Reid, G., Gibson, G.R., Merenstein, D.J., Pot, B., Morelli, L., Canani, R.B., Flint, H.J., Salminen, S., Calder, P.C. and Sanders, M.E., 2014. Expert consensus document. The International Scientific Association for Probiotics and Prebiotics consensus statement on the scope and appropriate use of the term probiotic. Nature Reviews Gastroenterology and Hepatology 11: 506-514. 
Hughes, S.A., Shewry, P.R., Gibson, G.R., McCleary, B.V. and Rastall, R.A., 2008. In vitro fermentation of oat and barley derived betaglucans by human faecal microbiota. FEMS Microbiology and Ecology 64: 482-493.

Kalliomaki, M., Salminen, S., Arvilommi, H., Kero, P., Koskinen, P. and Isolauri, E., 2001. Probiotics in primary prevention of atopic disease: a randomised placebo-controlled trial. The Lancet 357: 1076-1079.

Kankainen, M., Paulin, L., Tynkkynen, S., Von Ossowski, I., Reunanen, J., Partanen, P., Satokari, R., Vesterlund, S., Hendrickx, A.P., Lebeer, S., De Keersmaecker, S.C., Vanderleyden, J., Hamalainen, T., Laukkanen, S., Salovuori, N., Ritari, J., Alatalo, E., Korpela, R., Mattila-Sandholm, T., Lassig, A., Hatakka, K., Kinnunen, K.T., Karjalainen, H., Saxelin, M., Laakso, K., Surakka, A., Palva, A., Salusjarvi, T., Auvinen, P. and De Vos, W.M., 2009. Comparative genomic analysis of Lactobacillus rhamnosus GG reveals pili containing a human-mucus binding protein. Proceedings of the National Academy of Sciences of the USA 106: 17193-17198.

Kelesidis, T. and Pothoulakis, C., 2012. Efficacy and safety of the probiotic Saccharomyces boulardii for the prevention and therapy of gastrointestinal disorders. Therapeutic Advances in Gastroenterology 5: 111-125.

MacDonald, E., Moller, K.E., Wester, A.L., Dahle, U.R., Hermansen, N.O., Jenum, P.A., Thoresen, L. and Vold, L., 2015. An outbreak of enterotoxigenic Escherichia coli (ETEC) infection in Norway, 2012: a reminder to consider uncommon pathogens in outbreaks involving imported products. Epidemiology and Infection 143: 486-493.

Macfarlane, G.T. and Englyst, H.N., 1986. Starch utilization by the human large intestinal microflora. Journal of Applied Bacteriology 60: 195-201.

Manners, D.J., Masson, A.J. and Patterson, J.C., 1973. The structure of a beta-(1 leads to 3)-D-glucan from yeast cell walls. Biochemical Journal 135: 19-30.

Moens, F., Verce, M. and De Vuyst, L., 2017. Lactate- and acetate-based cross-feeding interactions between selected strains of lactobacilli, bifidobacteria and colon bacteria in the presence of inulin-type fructans. International Journal of Food Microbiology 241: 225-236.

Moré, M.I. and Swidsinski, A., 2015. Saccharomyces boulardii CNCM I-745 supports regeneration of the intestinal microbiota after diarrheic dysbiosis - a review. Clinical and Experimental Gastroenterology 8: 237-255.

Pakalniskiene, J., Falkenhorst, G., Lisby, M., Madsen, S.B., Olsen, K.E., Nielsen, E.M., Mygh, A., Boel, J. and Molbak, K., 2009. A foodborne outbreak of enterotoxigenic E. coli and Salmonella Anatum infection after a high-school dinner in Denmark, November 2006. Epidemiology and Infection 137: 396-401.

Pena, J.A. and Versalovic, J., 2003. Lactobacillus rhamnosus GG decreases TNF-alpha production in lipopolysaccharide-activated murine macrophages by a contact-independent mechanism. Cellular Microbiology 5: 277-285.

Pessi, T., Sütas, Y., Hurme, M. and Isolauri, E., 2000. Interleukin-10 generation in atopic children following oral Lactobacillus rhamnosus GG. Clinical and Experimental Allergy 30: 1804-1808.
Pop, M., Paulson, J.N., Chakraborty, S., Astrovskaya, I., Lindsay, B.R., Li, S., Bravo, H.C., Harro, C., Parkhill, J., Walker, A.W., Walker, R.I., Sack, D.A. and Stine, O.C., 2016. Individual-specific changes in the human gut microbiota after challenge with enterotoxigenic Escherichia coli and subsequent ciprofloxacin treatment. BMC Genomics 17: 440.

Qadri, F., Svennerholm, A.M., Faruque, A.S. and Sack, R.B., 2005. Enterotoxigenic Escherichia coli in developing countries: epidemiology, microbiology, clinical features, treatment, and prevention. Clinical Microbiology Reviews 18: 465-483.

Raybaudi-Massilia, R.M., Mosqueda-Melgar, J., Soliva-Fortuny, R. and Martín-Belloso, O., 2009. Control of pathogenic and spoilage microorganisms in fresh-cut fruits and fruit juices by traditional and alternative natural antimicrobials. Comprehensive Reviews in Food Science and Food Safety 8: 157-180.

Roselli, M., Finamore, A., Britti, M.S. and Mengheri, E., 2006. Probiotic bacteria Bifidobacterium animalis MB5 and Lactobacillus rhamnosus GG protect intestinal Caco-2 cells from the inflammation-associated response induced by enterotoxigenic Escherichia coli K88. British Journal of Nutrition 95: 1177-1184.

Roussel, C., Cordonnier, C., Livrelli, V., Van de Wiele, T. and Blanquet-Diot, S., 2017a. Enterotoxigenic and enterohemorrhagic Escherichia coli: survival and modulation of virulence in the human gastrointestinal tract. Escherichia coli: recent advances on physiology, pathogenesis and biotechnological applications. InTechOpen, London, UK.

Roussel, C., Sivignon, A., De Vallee, A., Garrait, G., Denis, S., Tsilia, V., Ballet, N., Vandekerckove, P., Van de Wiele, T., Barnich, N. and Blanquet-Diot, S., 2018. Anti-infectious properties of the probiotic Saccharomyces cerevisiae CNCM I-3856 on enterotoxigenic E. coli (ETEC) strain H10407. Applied Microbiology and Biotechnology 102: 6175-6189.

Roussel, C., Sivignon, A., De Wiele, T.V. and Blanquet-Diot, S., 2017b. Foodborne enterotoxigenic Escherichia coli: from gut pathogenesis to new preventive strategies involving probiotics. Future Microbiology 12: 73-93.

Salimian, J., Salmanian, A., Khalesi, R., Mohseni, M. and Moazzeni, S., 2010. Antibody against recombinant heat labile enterotoxin B subunit (rLTB) could block LT binding to ganglioside M1 receptor. Iranian Journal of Microbiology 2: 120-127.

Shin, J., Yoon, K.B., Jeon, D.Y., Oh, S.S., Oh, K.H., Chung, G.T., Kim, S.W. and Cho, S.H., 2016. Consecutive outbreaks of enterotoxigenic Escherichia coli O6 in schools in South Korea caused by contamination of fermented vegetable kimchi. Foodborne Pathogens and Disease 13: 535-543.

Stratford, M. and Eklund, T., 2003. Organic acids and esters. In: Russell, N.J. and Gould, G.W. (eds.) Food preservatives. Springer US, Boston, MA, USA, pp. 48-84.

Szajewska, H., Wanke, M. and Patro, B., 2011. Meta-analysis: the effects of Lactobacillus rhamnosus GG supplementation for the prevention of healthcare-associated diarrhoea in children. Alimentary Pharmacology and Therapeutics 34: 1079-1087. 
Taniuchi, M., Walters, C.C., Gratz, J., Maro, A., Kumburu, H., Serichantalergs, O., Sethabutr, O., Bodhidatta, L., Kibiki, G., Toney, D.M., Berkeley, L., Nataro, J.P. and Houpt, E.R., 2012. Development of a multiplex polymerase chain reaction assay for diarrheagenic Escherichia coli and Shigella spp. and its evaluation on colonies, culture broths, and stool. Diagnostic Microbiology and Infectious Disease 73: 121-128.

Tompkins, T.A., Mainville, I. and Arcand, Y., 2011. The impact of meals on a probiotic during transit through a model of the human upper gastrointestinal tract. Beneficial Microbes 2: 295-303.

Tsai, C.-C., Lin, P.-P. and Hsieh, Y.-M., 2008. Three Lactobacillus strains from healthy infant stool inhibit enterotoxigenic Escherichia coli grown in vitro. Anaerobe 14: 61-67.

Van den Abbeele, P., Roos, S., Eeckhaut, V., MacKenzie, D.A., Derde, M., Verstraete, W., Marzorati, M., Possemiers, S., Vanhoecke, B., Van Immerseel, F. and Van de Wiele, T., 2012. Incorporating a mucosal environment in a dynamic gut model results in a more representative colonization by lactobacilli. Microbial Biotechnology 5: 106-115.

Van den Broek, M.F.L., De Boeck, I., Claes, I.J.J., Nizet, V. and Lebeer, S., 2018. Multifactorial inhibition of lactobacilli against the respiratory tract pathogen Moraxella catarrhalis. Beneficial Microbes 9: 429-439.

Vanderhoof, J.A., Whitney, D.B., Antonson, D.L., Hanner, T.L., Lupo, J.V. and Young, R.J., 1999. Lactobacillus GG in the prevention of antibiotic-associated diarrhea in children. The Journal of Pediatrics 135: 564-568
Voreades, N., Kozil, A. and Weir, T.L., 2014. Diet and the development of the human intestinal microbiome. Frontiers in Microbiology 22: 494 .

Wang, T., Teng, K., Liu, G., Liu, Y., Zhang, J., Zhang, X., Zhang, M., Tao, Y. and Zhong, J., 2018. Lactobacillus reuteri HCM2 protects mice against enterotoxigenic Escherichia coli through modulation of gut microbiota. Scientific Reports 8: 17485.

Youmans, B.P., Ajami, N.J., Jiang, Z.D., Campbell, F., Wadsworth, W.D., Petrosino, J.F., DuPont, H.L. and Highlander, S.K., 2015. Characterization of the human gut microbiome during travelers' diarrhea. Gut Microbes 6: 110-119.

Zhang, L., Xu, Y.Q., Liu, H.Y., Lai, T., Ma, J.L., Wang, J.F. and Zhu, Y.H., 2010. Evaluation of Lactobacillus rhamnosus GG using an Escherichia coli K88 model of piglet diarrhoea: effects on diarrhoea incidence, faecal microflora and immune responses. Veterinary Microbiology 141: 142-148.

Zhang, W., Zhu, Y.-H., Zhou, D., Wu, Q., Song, D., Dicksved, J. and Wang, J.-F., 2017. Oral administration of a select mixture of Bacillus probiotics affects the gut microbiota and goblet cell function following Escherichia coli challenge in newly weaned pigs of genotype muc4 that are supposed to be enterotoxigenic E. coli f4ab/ac receptor negative. Applied and Environmental Microbiology 83: e02747-02716.

Zott, K., Claisse, O., Lucas, P., Coulon, J., Lonvaud-Funel, A. and Masneuf-Pomarede, I., 2010. Characterization of the yeast ecosystem in grape must and wine using real-time PCR. Food Microbiology 27: 559-567. 
